\title{
THE OCARECLOUDS PROJECT: TOWARDS ORGANIZING CARE through trusted Cloud Services
}

Femke De Backere ${ }^{1}$, Femke Ongenae ${ }^{1}$, Frederic Vannieuwenborg ${ }^{1}$, Jan Van Ooteghem ${ }^{1}$, Pieter Duysburgh ${ }^{2}$, Arne Jansen ${ }^{3}$, Jeroen Hoebeke ${ }^{1}$, Kim Wuyts $^{4}$, Jen Rossey ${ }^{1}$, Floris Van den Abeele $^{1,}$ Karen Willems ${ }^{2}$, Jasmien Decancq ${ }^{2}$, Jan Henk Annema ${ }^{3}$, Nicky Sulmon ${ }^{3}$, Dimitri Van Landuyt $^{4}$, Stijn Verstichel ${ }^{1}$, Pieter Crombez ${ }^{5}$, Ann Ackaert $^{1}$, Dirk De Grooff ${ }^{3}$, An Jacobs ${ }^{2}$ and Filip De Turck ${ }^{1}$

${ }^{1}$ Information Technology Department (INTEC), Ghent University - iMinds, Gaston Crommenlaan 8, bus 201, 9050 Ghent, Belgium

${ }^{2}$ Research Centre for Studies on Media, Information and Telecommunication (SMIT), Brussels University (VUB) iMinds, Pleinlaan 2, 1050 Brussels, Belgium

${ }^{3}$ Centre for User Experience Research (CUO), KU Leuven - iMinds, Parkstraat 45, bus 3605, 3000 Leuven, Belgium

${ }^{4}$ Distrinet Research Group, KU Leuven - iMinds, Celestijnenlaan 200A, 3001 Heverlee, Belgium

${ }^{5}$ Televic Healthcare NV, Leo Bekaertlaan 1, 8870 Izegem, Belgium

\section{ABSTRACT}

The increasing elderly population and the shift from acute to chronic illness, makes it difficult to care for people in hospitals and rest homes. Moreover, elderly people, if given a choice, want to stay at home as long as possible. In this paper, the methodologies to develop a cloud-based semantic system, offering valuable information and knowledge-based services are presented. The information and services are related to the different personal living hemispheres of the patient, namely the daily care related needs, the social needs and the daily life assistance. Ontologies are used to facilitate the integration, analysis, aggregation and efficient use of all the available data in the cloud. By using an interdisciplinary research approach, where user researchers, (ontology) engineers, researchers and domain stakeholders are at the forefront, a platform can be developed of great added value for the patients that want to grow old in their own home and for their caregivers.

\section{KEYWORDS}

eCare, cloud, trust, ontologies, interdisciplinary 


\section{CORRESPONDENCE TO:}

\section{Femke De Backere}

Department of Information Technology

Internet Based Communication Networks and Services (IBCN)

Ghent University - iMinds

Gaston Crommenlaan 8 (Bus 201), B-9050 Gent, Belgium

T: +32 93314938

F: +32 93314899

E: femke.debackere@intec.UGent.be 


\section{INTRODUCTION}

The increasing elderly population in most industrialized countries will lead to many challenges in the upcoming years. Simultaneously, there is also a shift in the burden of illness from acute to chronic conditions. This drives up health costs and creates a generation of people living with long-term illness and disability. Providing good quality of care to more elderly and chronically ill people, with a lower availability of professional caregivers and within a stringent healthcare budget calls for innovative solutions.

A trend in healthcare towards resolving these challenges is a move towards increased home care. This is intended to keep patients at home and give them outpatient care as long as possible. Therefore, there is an increasing demand for services that will facilitate an elongated stay at home. To realize this, cost-efficient and qualitative care solutions at home should be available. Information technology (IT) has a considerable potential to improve the delivery and quality of care in the home environment (referred to as eCare) [1] and can stimulate smart product and service concepts in the setting of the patient's home in order to reduce social exclusion. The introduction of these services also affects the operational care processes in a positive manner, e.g., more efficient patient visits, improved communication, better follow-up and a decrease of work pressure.

eCare is typically confronted with a double challenge. On the one hand, patients have the need for an integrated service, in which personalized services are offered and where content is adapted to the needs of each individual [2]. On the other hand, there is the need for interaction and the efficient cooperation of a plethora of caregivers. These caregivers range from formal ones, e.g., general practitioners and nurses, to informal ones, e.g., family, housekeepers and neighbors. A huge amount of diverse information is gathered by these different actors, ranging from information concerning the daily activities of the patient in view of their personal health to feelings of security and social inclusion. Providing the adequate information to the health provider at the appropriate time and in the appropriate place should be able to improve the care process, lower the risk of medical errors and assist in lowering the overall cost.

Several IT platforms exist to support the formal caregivers in the collection of this data. However, each organization tends to use its own platform, which limits the data exchanging 
possibilities. Moreover, informal caregivers are usually excluded from these systems, resulting in parallel communication via notes, post-its and booklets. Thus, the huge amount of interesting data, created for daily care, is not used in an integrated and intelligent way, forcing the caregivers to process the same data more than once.

As indicated by Koch [2], this will require a cross-disciplinary and interdisciplinary approach, as social, technical and medical needs of the ageing individuals have to be considered and taken into account. eCare is confronted with challenges that are strongly related to technology, human characteristics, but also the socioeconomic context [3], this requires a holistic approach: the success of a eCare technology depends on taking these different aspects and their interrelatedness into account throughout the whole development process of the technology.

Both eHealth -and eCare services face several barriers that hinder their general deployment and social uptake. eHealth services are services for supporting, automating and facilitating medical treatments and/or processes such as teledermatology, telemedicine, hospital information systems (HIS), while eCare services have a strong focus on the improvement of the care process and personal wellness of patients and caregivers, e.g. video calling and fall detection. In general, following topics can be pointed out as the real obstacles [4]. First, the actual financing models for care and cure often do not allow real changes in the task descriptions of several actors, let alone innovations in the care process. Second, depending on the eHealth or eCare service, the current set of regulations is deficient and needs to be extended and adopted to allow innovations in healthcare delivery. Third, together with the growing interest in IT-supported care and cure services, also the need for common data standards increases further. Fourth, often the added value of services is not clear. Without a clear view on the added value and the absence of a viable financing model, not all caregivers are that enthusiastic on eHealth and eCare services. Finally, often patients are not informed (enough) about the possibilities of care innovation. Moreover, most patients adopt a passive health consuming role instead of active participation in their own healthy life.

The costs for health services are rising for patients as well as care organizations. While adopting IT can mean a decrease in these costs, it is also of great importance that the hardware used for this adoption is well cared for and maintained [5]. This in itself can be a 
very expensive operation, as software and data needs to be backed-up and personnel with IT expertise should be hired to set-up and maintain these systems. Moreover, the complexity of these systems will grow in the coming years, as there is a shift from passive systems to more interactive systems, with a focus on the quality of care regarding the patient [6]. Cloud computing provides another solution for these "local" systems that could be used to manage the system of only one healthcare organization. By using cloud computing, resources are shared, this means that only the necessary resources are used and thus that the expenses are lower than managing and maintaining a local system. Adopting cloud technology also shifts the burden from management and maintenance of the IT developers and administrators to the development of specific healthcare services that can make the difference. It is also easier to integrate data that is originating from different sources in the cloud.

Also, governments are setting up new initiatives for in practice information sharing, for example the eHealthPlatform and Vitalink [7][8]. The eHealthPlatform is an official federal network in Belgium used to exchange health data for many applications. Vitalink ${ }^{1}$, a Flemish initiative, was developed for data sharing, with an emphasis on improving the cooperation and organization of health records. Moreover, Vitalink focuses on offering a secure way to access this information based on trust relationships between patients and caregivers. This additional information can be used to provide better care for patients, for example, Vitalink provides up to date information concerning the medication scheme of patients. Such platforms offer data in a decentralized manner, while they ensure that the data kept within their databases is the most up-to-date information at a given time.

In this paper, a cloud-based semantic platform is presented, namely as researched in the iMinds - interdisciplinary - project OCareCloudS (OCCS) [9], which allows for offering information and knowledge-based services, crossing the border of different personal living hemispheres of the patient, namely the daily care related needs, the social needs and the daily life assistance. This platform is able to integrate the heterogeneous information about a patient made available through the various sources and actors by using an ontology-based approach. By using a cloud-based solution, there can be a shift from management and

\footnotetext{
${ }^{1}$ http://www.vitalink.be/Vitalink/Over-Vitalink/Over-Vitalink/
} 
maintenance of a platform to offering better services. Moreover, by offering a cloud solution, data from different care organizations can be integrated, as often patients receive care from different organizations. It also makes it easier to incorporate data that will become readily available in the future by new initiatives of governments. Finally, costs will be reduced as only the necessary resources are used. Within the project, we have strongly focused on the security of the data of the patients, informal and formal caregivers. Due to paper length limitations, we refer to future publications for details about the specific security implementations of the platform.

Additionally, the OCCS system offers solutions for the trustworthy creation of care networks and a user-centric control by the patient. The access to the OCCS system and its services is thus based on trust, allocated by the patient to the actual personal network of formal and informal caregivers. This allows for a higher involvement of the informal caregivers and ensures that they can easily participate in this platform, if trust is allocated to them by the patient. Moreover, this platform also focuses on facilitating and improving the quality of life for the patient (and not only the quality of care). To achieve this, a user-centered approach is followed throughout the entire research and development process. First of all, patients and other actors in their care network are observed to fuel the domain analysis. Secondly, the vast amount of data in the domain analysis is translated into communication tools that help the development team to keep the users in mind at all times. Finally, workshops and individual sessions are organized with stakeholders to evaluate and co-create the OCareCloudS platform. In this context, stakeholders are the companies and organizations that are interested in using the provided solution, whereas actors are the (in)formal caregivers, patients and other users utilizing the provided system. After this initial user research process and during the iterative repetition of the process, technological research questions did arise. How can we integrate all kinds of data, ranging from information in the heads of the (in)formal caregivers to data, made available by sensors and other data sources. Moreover, how can a cloud-based solution be developed, that can easily be extended by new services to the platform?

The remainder of the paper is organized as follows. Section 2 elaborates on the methodology used during the project. Section 3 focuses on the user and domain analysis, while Section 4 details the architecture of the OCCS system. Section 5 reveals the origin of 
the data used. Finally, the conclusions are discussed in Section 6.

\section{Methodology}

As detailed in the introduction, the creation of a platform that facilitates management of daily home care by integrating a multitude of data, is by no means an easy undertaking and requires a holistic approach in order to ensure the uptake of the technology.

When looking at technical aspects, the OCCS system will make use of ontology engineering to manage the data, information and knowledge available in the cloud. The construction of such an ontology is a delicate undertaking. The home care domain needs to be reflected in the ontology, this requires a very thorough understanding of the domain and which aspects of the domain can be formalized in an ontology and which can not.

This means that there is a large dependency on the input from user researchers. A thorough analysis of the domain has to serve as the basis on which the continuous care ontology will be extended. Van Gemert-Pijnen et al. [3] also refer to contextual inquiry as important to understand how technology can match the current working habits of the targeted users. The user research is required to identify the needs of both caregivers and patients with regard to the daily management of home care, as this will improve the level of acceptance and adoption [10]. As such, it should become more clear what services should actually be offered to the patient and the network of caregivers surrounding the patient. These services need to be developed carefully, in order for them to offer sufficient added value for the targeted users. Also, while these services might disrupt current work practices, they nevertheless need to take into account the structure of a domain and the current work practices. An understanding of the context and the user is elementary to anticipate issues that impede user adoption of the technology as much as possible.

From a market perspective, an increasing amount of stakeholders involved in home care also poses challenges in relation to the business model and the value network analysis that was carried out in scope of the OCCS project, but is not explained in detail in this paper. Many eHealth and eCare services have been developed in recent years, but their deployment is often troubled and very few of them have resulted in actual viable market solutions. There is a need for more clarity regarding which actor takes up which role, what the added value is 
for each actor involved and a clear quantitative and qualitative impact analysis of the considered/envisioned service. In order to develop eHealth and eCare services that are supported by the involved actors and imbedded in the care and cure processes, frameworks that highlight and detect collaborations or conflicts in the business models for each individual actor involved in the service provisioning are needed. Limburg et al. [10] describe the need of business modeling with all involved actors for deploying eHealth technologies in a sustainable way. The CeHRes Roadmap [10] guides the eCare or eHealth service developers and the involved actors through the collaboration and co creation development process. This value-driven approach could lead to a more successful integration and implementation of the services. At last, the methodology of Vannieuwenborg et al. [11] describes a multi-actor evaluation of the impact of eCare services. The model allows determining conflicts in the business models of the involved actors and provides possible strategies to dissolve the issues.

To coordinate the activities in the OCCS project, the consortium uses a so-called 'innovation binder' that integrates the infrastructural requirements of the OCCS system with the user needs that result from the domain analysis and link these with the value network analysis. The innovation binder (see Section 3.2) is a central tool in the project, in order to facilitate the development of the proof-of-concept and the integration work needed to realize it.

Van Gemert-Pijnen et al. [3] have depicted the holistic development process as consisting of multidisciplinary project management, contextual inquiry, value specification, design, operationalization and summative evaluation. As will become clear in the following paragraphs, most of these principles are applied in the OCCS project. The 'innovation binder' method or tool has been created precisely for facilitating the multidisciplinary project management. Much of the user research consists of making a contextual inquiry and iterative testing of the design with users, by means of mock-ups and paper prototypes. Moreover, one of the specific innovation binder activities is determining how functionalities of the eHealth technology relate to the values of all stakeholders in the project. However, while we agree with Van Gemert-Pijnen et al. that the actual deployment and evaluation of the actual uptake of the technology should ideally also be part of the development process, the OCCS project is a research project, and therefore we were unable to make these activities part of the development of the OCCS system. 


\section{USER AND DOMAIN ANALYSIS}

The continuous care ontology has to integrate the huge amounts of data relevant for daily home care and has to describe all the concepts in the domain in detail. There are several methodologies for ontology engineering and there is a considerable variation in the degree in which they include domain experts in building the knowledge model. Some methodologies tend to emphasize the role of the knowledge engineer, such as the Tove, Enterprise and Methontology approach [13], while other methodologies, e.g., HCOME [14], tend to rely on domain experts and require them to make a considerable time-investment to construct the ontology themselves.

In the iMinds ACCIO project, middle ground was found between these two opposed approaches, in which domain experts (caregivers and patients) are involved in each step of the ontology creation [15]. The OCCS project takes a similar approach, which consists of an extensive domain analysis first, followed by a series of workshops.

The role of user researchers in this process is to facilitate a process of 'mutual learning' between user and designer or developer [16]. User researchers need to understand the domain as well as the targeted users and make this knowledge accessible to developers and engineers.

The participating patients and caregivers approved to be part of this study by signing an informed consent, giving permission to use the acquired data and information for scientific purposes on the condition that confidentiality is ensured. This project is also registered with the Commission for the Protection of Privacy (CPP) ${ }^{2}$ as an iMinds ICON project. The study was exempt from formal ethical approval.

\subsection{DOMAIN ANALYSIS}

An extensive domain analysis was needed at the start of the project, for (1) the extension of the continuous care ontology, (2) the identification of all the stakeholders in the value network (3) the identification of stakeholder (patients and caregivers) needs with relation to daily home care management in order to determine what services should be provided by the

\footnotetext{
${ }^{2}$ http://www.privacycommission.be/en
} 
platform.

In order to achieve this, a team of user researchers did a domain analysis, combining desk research, with expert interviews and observations to gain a comprehensive view of the home care domain. The observations included a contextual inquiry with a large number of primary and secondary stakeholders. Two care organizations were involved in the project: a home care organization and a care organization that offers other types of care services (meal deliveries, cleaning, institutional care). Several types of employees of these organizations were observed and interviewed about their work, how they collaborated with other caregivers and the issues they were professionally faced with. Through these participative observations, the researchers also got to visit patients and their informal caregivers at home. Informal caregivers were also contacted through non-profit organizations advocating their cause.

The result of this large undertaking was an elaborated report, giving its readers a good understanding of the insights of the various stakeholders involved in home care, the care data they make use of and the information flow between them. These insights were also gathered in an extensive mind map that describes all roles that are involved in home care, the tasks associated with each role, how the person taking on the role is collaborating with other roles and which tools are used for collaboration. In Figure 1, an example of the structure of the mind map is visualized. Once it was constructed, the mind map was validated with the domain experts. The mind map served as input for the ontology engineers, working on the extension of the continuous care ontology. The domain analysis served to identify the needs of the targeted users, which served as a starting point for the services that need to be offered on the platform.

\section{2 'InNovation Binder’ activities: Persona \& SCEnARIO DEVElopment}

For the innovation binder to serve as a coordinating tool in the project, the results of the domain analysis had to become part of it. In order to make the bulk of results of the domain analysis easily accessible for the project team, a number of personas were created. Personas are often created in the design process to represent the problems and needs of the target group [17]. They represent a kind of 'archetypical' user, helping project team members to understand about what it would be like to belong to the target group, to always keep the 
end user in mind during the entire development process. Eight personas were created in an early stage of the project, after finishing up the domain analysis. The personas consisted of four patients and four formal caregivers. The informal caregivers of the patients were included in the personas of the patients themselves. These personas were discussed with the care organizations involved in the project and adapted. Some characteristics were made more realistic and separate personas were created for informal caregivers. Finally, this resulted in four patients (of which one also takes on the role of informal caregiver), four formal caregivers and three additional informal caregivers. They are visualized on posters with an image and a short text explaining their (care) situation, personality, wishes and needs. These posters were hung in sight during meetings to more easily refer to the personas and to always remember them.

In addition to these personas, the needs, or 'challenges', identified in the domain analysis were listed, and together these were used as the basis for creativity sessions on the needed services. These creativity sessions were organized using the 'relay ideation method' [18]. This brainstorm method specifically helped the project team to move from the domain analysis towards thinking about solutions for the needs and challenges identified in the domain analysis. The 'relay ideation method' requires all participants to first gain more empathy with the targeted end users. In this case, all participants were asked to read the personas. Next, they were given a 'challenge', and by making use of so-called 'concept sheets' all participants were involved in a process that in a stepwise manner moved the participants from thinking about the current state towards future oriented thinking.

Next, these concepts were reviewed by the user researchers and served as inspiration for the future scenarios, which detailed how the personas interact with a possible OCCS system. In an initial version, the services provided on the OCCS system were described on a conceptual level. These user scenarios were then presented to the project consortium for feedback on eligibility, feasibility and novelty. With this feedback in mind, a new iteration of the scenarios was written. These scenarios were then used to evaluate the initially planned architecture of the OCCS system and to decide what features to develop in (the different iterations of) the proof of concept.

\subsection{CO-DESIGN SESSIONS: ITERATIVE SERVICE DEVELOPMENT}


In the next steps of the ongoing user research, we will further go into the specific services that were presented in the scenarios and the implications they have in their lives. All services bring up issues and questions on which user input is needed. However, this input does not only have impact on the type of services, but could have more fundamental technical implications. Insights gained throughout these workshops may require the ontology engineers to adjust the continuous care ontology.

A first type of sessions that will be set-up, will be the presentation of the scenarios to the targeted users (both caregivers and patients). These scenarios will be visualized and made more easily comprehendible so that they serve as a good basis to discuss the OCCS system and its services. In subsequent workshops, more detailed elements in the scenarios that require more attention from a user side will be further explored.

\subsection{Persona \& SCEnARIO OUtLine}

The following subsections focus on presenting some of the personas and a scenario, developed during the Innovation Binder activities. The personas and scenarios gave an interesting viewpoint on how all actors and stakeholders see the innovation that is necessary in the eCare domain.

\subsubsection{PERSONAS}

Yousuf, an 80 years old patient, lives with his daughter Fatima since the death of his wife 8 years ago. Due to his unhealthy lifestyle, he is obese and lies in the sofa most of the day. As a result, he developed a few bedsores, requesting much attention of a nurse. Yousuf has no strong relationship with the family of his daughter and often feels lonely. Not long ago, Yousuf has fallen and has increased mobility problems ever since. He feels isolated in his own private rooms.

Fatima, 44 years old, has converted the garage into a bedroom and living room, which enables her to take care of her father while he lives with her and her family. Fatima has 4 children and has requested help of formal caregivers, because the additional care required for her father causes a heavy burden in combination with her other daily and professional activities.

Lydia is the formal caregiver of Yousuf. Karen, a colleague, will replace her if necessary. Eline 
is a nurse, working for a rest home in the neighborhood. Anne is a neighbor and helps out when Yousuf or Fatima need help and no one else can assist them.

\subsubsection{SCENARIO}

Every morning, just before setting of for work, Fatima helps Yousuf out of bed. She checks on him during the day whenever she has the possibility. Fatima has installed the OCCS application on her smartphone and she has indicated through the settings that Yousuf should be out of bed every day by 10 o'clock and that she wants to be kept informed of any changes made to/by the system.

After Fatima helped Yousuf out of bed on Monday, she looks in the fridge to make sure there is still sufficient food. Fatima estimates that some groceries need to be done for Yousuf for coming days. She mentions to Yousuf that she will do the shopping in the evening. As she is in a hurry and leaves for work.

Later that day, when Fatima wants to go shopping, she receives a phone call. Her son has had an accident during football practice and she has to bring him to the hospital. She decides to enter the task into the OCCS system through the application on her smartphone. At home, Yousuf receives a notification of the shopping task on his TV. This way, he is able to check whether the attached shopping list is correct and he is capable of adding other items to the list.

Tuesday morning, Lydia checks if she has any new tasks. She consults the list on her smartphone and notices the request to do some groceries. She confirms that she will take up this task. This triggers a notification being sent to Fatima, which makes her feel at ease.

On Wednesday, Fatima is on an early shift and has arranged that Anne, the neighbor, will help Yousuf out of bed. However, due to some misunderstanding, Anne has forgotten to do so. However, for his own comfort, Yousuf's bed is equipped with sensors that register if he is lying in bed. At 10 o'clock, the OCCS system is triggered because he is still in bed and Ann has forgotten to help him. The OCCS will send a message to Yousuf, asking if somebody should be notified to help him out of bed. Yousuf indicates that he should be helped out of bed. The system knows that Anne was responsible for executing this task and notifies her that she has forgotten about Yousuf. Unfortunately, Anne is not in the neighborhood and thus is not 
capable of helping Yousuf. She feels silly for forgetting about Yousuf and has to decline the notification. The OCCS system determines the next (in)formal caregiver that can be notified to handle the task, based on the current situation. The system decides that Eline should be contacted, as she works nearby and Fatima is unable to make it as she is at work.

Eline is notified by the system with the request to help Yousuf out of bed. She accepts the task.

\section{ARCHITECTURE OF THE OCCS SYSTEM}

The OCCS system offers a way to integrate all available data of a patient in one platform. Section 4.1 details how data is transformed into information that can be used by the system, an overview of the different transformations and acronyms can be found in Figure 2. As a result of the user research, the OCCS system's architecture was developed, visualized in Figure 3, Within this architecture, the Controllers, the OCarePlatform and the Datasources all run in the cloud, which can either be a private or public cloud. These components are not necessarily maintained in the same cloud. For example, the Controllers can be run in a public cloud, while the OCarePlatform operates in a private cloud. The Datasources can be resources of the OCCS project or external resources, for example Vitalink. The different acronyms at the arrows are used to indicate what data type is sent to the OCarePlatform. As data works its way through the OCCS System, it will be enriched and altered in order to enable the processing of the data by the OCarePlatform. Section 0 discusses the internal functioning of the OCarePlatform. This platform is responsible for reasoning on the data that it receives and based on this information sends alerts, notifications and suggestions to its users. A high-level overview of the OCarePlatform is detailed in Figure 4.

\subsection{From Care Data to KnOWLEdge}

The OCCS system aims to gather and integrate the available information about a patient. The data fed to the OCCS system will originate from different sources, including input provided by (in)formal caregivers, data from existing platforms or data generated by devices and applications in the patients' residence. 
As it is difficult to process raw data, or Care Data (CD), data originating from the patient residence will be collected on the terminal. There, in a first phase, data will be transformed into "Meta Care Data" (MCD). MCD is Care Data, which is enriched with information about the patient and linked with other specific information, e.g., timestamps. Next, MCD is transformed into "Meta Care Information" (MCl). $\mathrm{MCl}$ is in fact MCD that is tagged. MCD is tagged with one or more "Meta Care Concepts" (MCC). These concepts can be found in the ontology used by the OCarePlatform. This way, the OCarePlatform knows how the CD, the most essential information, can be mapped onto the ontology. By providing data conforming to the ontology, the system is able to reason on this available data.

$\mathrm{MCl}$ is sent to the Controllers, this is a server-side system, managing the connection between the different clients and the OCarePlatform. Its main function is managing the data sent from a patient or the caregiver to the OCarePlatform and vice versa. The Controllers are also responsible for handling back-ups and storing the data.

The reasoning process, within the OCarePlatform, creates new knowledge, called "Meta Care Knowledge" (MCK), that is inferred out of a combination of $\mathrm{MCl}$ and/or $\mathrm{MCK}$.

\subsection{OCAREPLATFORM}

To facilitate the intelligent and coordinated integration, analysis, combination and efficient usage of all the $\mathrm{MCl}$ available in the cloud, the OCarePlatform was designed. A closer view on the OCarePlatform is shown in Figure 4, This management system's task is to match the $\mathrm{MCl}$, provided by different personal care services, on services that are interested in related data. These latter services are called the Meta Care Information extraction services, or also $\mathrm{MCl}$ services.

The $\mathrm{MCl}$ services summarize and provide information to the (in)formal caregivers interested in certain topics. For example, the physical activity $\mathrm{MCl}$ service groups information about the physical activity of a patient provided by various devices, e.g., the domestics system monitoring movement in the home of the patient and the movement buddy counting the steps the patient takes. It combines this information to give a general idea of the physical activity of the patient.

Thus, for the $\mathrm{MCl}$ services to work properly, they need to be able to collect all the $\mathrm{MCl}$ 
provided by the personal care services related to their topic of interest. To facilitate the matching of $\mathrm{MCl}$ on $\mathrm{MCl}$ services, the OCarePlatform uses an ontology, which models all the data that can be communicated within the cloud. For example, the ontology contains the knowledge that data can pertain to a physical activity and that walking is a physical activity.

The OCarePlatform needs to match the various $\mathrm{MCl}$, provided by the personal care services and various other devices and sensors on the $\mathrm{MCl}$ services by using ontologies. Therefore, the Semantic Communication Bus (SCB) [19] will be used. It was developed to process data and forward it to applications that have indicated they have an interest for this type of data. The SCB orchestrates the communication of semantically enriched data. This allows filtering data based on meaning rather than on string patterns. The SCB interprets the data using a continuous care ontology, which models all the data that can be communicated within the cloud. The SCB allows components to subscribe to relevant $\mathrm{MCl}$, through the Context Disseminator.

The $\mathrm{MCl}$ services use a Context Interpreter, which manages the knowledge base and the associated semantic reasoning for deriving high-level context knowledge. Only a subset of the core ontologies, used by the SCB, are present to specify the context they are interested in. This is done by defining filtering rules and registering them with the Context Disseminator, through the Context Manager. These rules are expressed using MCC from the core ontologies. When $\mathrm{MCl}$ is published on the $\mathrm{SCB}$, the Context Disseminator will use a reasoner to match this information with the registered filter rules. If a match is found, the $\mathrm{MCl}$ is forward to the application components that are subscribed to the filter rule. The use of these filter rules thus reduces the amount of $\mathrm{MCl}$ that is forwarded to the $\mathrm{MCl}$ services. This prevents those components from being flooded with huge amounts of for them irrelevant context data, generated by the various sensors and devices connected to the platform. Filter rules can be dynamically added at any point of time by the $\mathrm{MCl}$ services. Performance and scalability are also optimized by using a subset of the core ontologies.

This way, $\mathrm{MCl}$ services will receive relevant context information. First, the OCarePlatform has to allow that the $\mathrm{MCl}$ services subscribe themselves to metadata they are interested in. This can be done through registration with MCC from the ontology, e.g., a physical activity $\mathrm{MCl}$ service subscribes to the physical activity $\mathrm{MCC}$ in the ontology. As a consequence, the 
software services will automatically receive all messages tagged with a tag that pertains to this knowledge. Second, the OCarePlatform also has to be able to pick up tagged data that is posted by the different personal care services. By performing reasoning on the ontology, the OCarePlatform will for example realize that walking is a physical activity and that physical activity $\mathrm{MCl}$ service will then receive all the messages form the movement buddy, because this device tagged its data with the tag walking.

The $\mathrm{MCl}$ services use ontologies, which model a specific domain to perform sophisticated reasoning. The applications can publish the conclusions on the SCB, through the Context Manager. These conclusions are called MCK. This information, in turn, can be picked up by other components to perform additional reasoning.

Another important component of the OCarePlatform, as visualized in Figure 4 , is the Big Mother Service (BMS). To allow advanced reasoning on the available $\mathrm{MCl}$ and $\mathrm{MCK}$ in the cloud, the BMS implements algorithms that reason on and combine $\mathrm{MCl}$ and $\mathrm{MCK}$ in the OCarePlatform to optimize the alerts given to the (in)formal caregivers. The BMS is in itself an intelligent software service. It implements rule-based algorithms that reason and combine $\mathrm{MCl}$ and $\mathrm{MCK}$ provided by the different $\mathrm{MCl}$ services and $\mathrm{MCl}$ originating from devices and sensors to optimize alerts given to the different (in)formal caregivers about the condition of a patient. A caregiver is able to indicate whether he of she wants to receive triggers based on $\mathrm{MCl}$ and MCK. For example, the caregiver can indicate that he or she wants to be notified if the activity of a person drops below a certain threshold. To achieve this, the BMS will periodically poll the physical activity $\mathrm{MCl}$ service to obtain the activity level of a person and match it to the specified threshold. If the threshold is crossed the BMS notifies the user immediately.

The service is called Big Mother, because in contrast with a big brother service, there is no decision internally on the value of the provided data. Everyone can contribute MCD and the decision on the value of the information produced by the $\mathrm{MCl}$ services and the suggested actions is given by "Big Mother" to the end users, checking on the trust relationship between its users guaranteeing a secure system. All these services will be offered to the stakeholders as a Software as a Service (SaaS) calling for a cloud approach in view of profiling, storage, scalability and semantics. 


\section{Generation of CARE DATA}

In order to extract as much knowledge as possible out of the Care Data and to be able to deliver value-added services, the platform supports Care Data coming from a variety of sources as briefly mentioned in Section 4.1. First of all, services will be provided to the (in)formal caregivers, which allow for submitting data through an application, running on the smartphone or tablet, or by sending a text message. These messages replace scribbles and notes in a classical booklet of the patient at home, which are difficult to manage and capture in the more formal eHealth and eCare platforms. Further, data collected from various existing eHealth and eCare platforms, which are already used by formal caregivers, will be fed to the system as well. How data from this application and from external existing platforms can be interpreted by the OCarePlatform is detailed in Section 5.1. Next to this, there is an important category of Care Data, pertaining to data coming from devices and applications in the residence of the patient. The data can be classified into two categories, namely "TV data", which is discussed in Section 5.2 and "sensor data", which is handled in Section 5.3.

\subsection{Data from Existing eHealth and eCARe Platforms}

The OCCS system is completed with a smartphone application for both formal and informal caregivers. Using this application, they can inject data into the OCCS system, which is automatically transformed into a format that is interpretable by the Controllers and the OCarePlatform.

Next to this, readily available data from other, existing platforms (e.g. information from the health and care organizations) can also be fed into the system by developing $\mathrm{MCl}$ services that are capable of transforming this platform specific data into $\mathrm{MCl}$. This can be a difficult process, but by adding this valuable, external information to the OCarePlatform, better decisions can be made. For instance, decisions can be taken based on the profile of the patient, the current circumstances and the work schedule of the caregivers of the health organizations. Another interesting example of data fed into the system originating from other data sources, is the medication scheme of patients. This information is available within 
the Vitalink platform ${ }^{3}$.

\subsection{TV DATA}

The current generation of care dependent people is in general not computer literate, which makes them digital outsiders from the current web applications. By choosing the TV as a well-known interaction surface, it enables these people to use digital services in an easy and safe way. With a simple dedicated remote control, the user can access the service of choice with a few steps. Such a system has been successfully demonstrated in [20], including services such as family pictures, music sharing, dissemination of information by care center, weather service, newspaper and Facebook. Through the patient's interaction with the TV, valuable Care Data can be generated such as the amount of time spent on TV-based services, most used services, service usage frequency. This can be further complemented with Care Data extracted from the TV device itself such as volume level, preferred channels.

\subsection{SENSOR DATA}

The other data is classified as "sensor data" and encompasses all other data extracted from the use of a heterogeneous set of personal devices in the patient's residence. In the OCareCloudS project, it is investigated how data coming from a large variety of Wi-Fi and IEEE 802.15.4 sensors and actuators deployed in a patient's residence can help to generate Care Data relevant to characterize the behavior and life of the elderly. For the integration of this sensor data in a Cloud-based platform, embedded web service technology based on the Constrained Application Protocol (CoAP) [21], which is being standardized, is used. CoAP is an application layer protocol designed for use in resource-constrained devices such as sensors. It is targeted to easily translate to HTTP for simplified integration with the web and uses the same RESTful principles as HTTP but with a much lower header overhead and parsing complexity than HTTP. As such, this is a perfect match to enable embedded devices to become providers of Care Data.

\section{CONCLUSION}

In this paper, the OCareCloudS project is presented. The methodology, used within the

\footnotetext{
${ }^{3}$ http://www.hziv.be/downloads/folder/vitalink.pdf
} 
project, actively involves user researchers, (ontology) engineers, techno-economic researchers and domain stakeholders, i.e. patients, informal caregivers as well as professionals working in the healthcare industry. One of the principle goals of the project is to support an elongated stay at home for elderly people and people with chronic disease, by offering optimized and individualized care at home and facilitating an increased collaboration between informal and formal caregivers. By involving all important stakeholders and actors in this interdisciplinary research, it was possible to capture the requirements, benefits and added value for patients and their caregivers. These outcomes, the personas and scenarios were used to develop a cloud-based solution, which is capable of increasing the uptake of the system and ensures that the platform offers services that are of real interest.

This approach enables improved care through better communication and information sharing, a reduction in cost and most importantly a higher involvement of the patient.

\section{ACKNOWLEDGMENT}

We would like to acknowledge that part of this research was supported by the iMinds Project OCareCloudS co-funded by the IWT, the iMinds and the following partners: Televic Healthcare, TPVision, Telecom IT and Boone NV. We would also like to thank OCMW-Kortrijk, OCMW-Ghent, Familiehulp for their cooperation in the project.

\section{CONFLICT OF INTEREST}

The authors declare that they have no conflict of interest. 


\section{REFERENCES}

[1] K. Gaßner and M. Conrad, "ICT enabled independent living for elderly: A status-quo analysis on products and the research landscape in the field of ambient assisted living (AAL) in EU-27," Institute for Innovation and Technology, VDI/VDE Innovation and Technik $\mathrm{GmbH}$, Berlin, Germany, Tech. Rep., March 2010.

[2] S. Koch, "Healthy ageing supported by technology a cross-disciplinary research challenge," Informatics for Health and Social Care, vol. 35, no. 34, pp. 81-91, Sep. 2010.

[3] J. van Gemert-Pijnen, N. Nijland, M. van Limburg, H. C. Ossebaard, S. M. Kelders, G. Eysenbach, and E. Seydel, "A holistic framework to improve the uptake and impact of eHealth technologies," Journal of Medical Internet Research, vol. 13, 2011.

[4] Ageing well in the Information Society: Action Plan on Information and Communication Technologies and Ageing [Internet]. European Commission; 2012 Dec 31 - [Cited 2013 Apr 30]; $\quad$ Available from: http://europa.eu/legislation_summaries/information_society/strategies//24292_en.htm

[5] AbuKhousa, Eman, Nader Mohamed, and Jameela Al-Jaroodi. "e-Health Cloud: Opportunities and Challenges." Future Internet 4.3 (2012): 621-645.

[6] Saranummi, N. "In the Spotlight: health information systems--mainstreaming mHealth." IEEE reviews in biomedical engineering 4 (2010): 17-19.

[7] France, Francis Roger. "eHealth in Belgium, a new "secure" federal network: Role of patients, health professions and social security services." International Journal of Medical Informatics 80.2 (2011): e12-e16.

[8] Van Cauter, Lies, Joep Crompvoets, and Joris Voets. "Intergovernmental information processes: an exploration of Flanders." status: published (2012).

[9] iMinds VZW [Internet]. OCareCloudS: Organizing Care with trusted, Cloud-like Services; 2012 - [Cited 2014 Jan 13]; Available from: http://www.iminds.be/en/research/overviewprojects/p/detail/OCareCloudS-2

[10]M. van Limburg, J. van Gemert-Pijnen, N. Nijland, H. C. Ossebaard, R. Hendrix, and E. Seydel, "Why business modeling is crucial in the development of eHealth technologies," Journal of Medical Internet research, vol. 13, 2011.

[11]F. Vannieuwenborg, J. van Ooteghem, M. Vandenberghe, S. Verbrugge, M. Pickavet, and D. Colle, "A methodology for multi-actor evaluation of the impact of eCare services,," presented at the SSH: IEEE International Workshop on Service Science for eHealth, Healtcom, 2013.

[12]S. Ballegaard, T. Hansen, and M. Kyng, "Healthcare in everyday life," in Proceeding of the twenty-sixth annual $\mathrm{CHI}$ conference on Human factors in computing systems - $\mathrm{CHI}$ '08. New York, NY, USA: ACM Press, 2008, pp. 1807-1816.

[13] H. Pinto and J. Martins, "Ontologies: How can they be built?" Knowledge and Information Systems, vol. 6, no. 4, pp. 441-464, Jul. 2004.

[14]K. Kotis and G. Vouros, "Human-centered ontology engineering: The HCOME methodology," Knowledge and Information Systems, vol. 10, no. 1, pp. 109-131, Jul. 2006.

[15]F. Ongenae, L. Bleumers, N. Sulmon, M. Verstraete, M. V. Gils, A. Jacobs, S. D. Zutter, P. Verhoeve, A. Ackaert, and F. D. Turck, "Participatory design of a continuous care ontology: towards a user-driven ontology engineering methodology," in KEOD 2011, Paris, France, Oct. 2011, pp. 81-90.

[16]T. Robertson and J. Simonsen, "Challenges and opportunities in contemporary participatory design," Design Issues, vol. 28, no. 3, pp. 3-9, Jul. 2012.

[17]J. Pruitt and J. Grudin, "Personas: practice and theory," in Proceedings of the 2003 conference on Designing for user experiences, ser. DUX '03. New York, NY, USA: ACM, 2003, pp. 1-15.

[18]K. Slegers, P. Duysburgh, and A. Jacobs. "The 'Relay Ideation' Technique: Moving from Problem Understanding to Problem Solving in the Design Process." Human-Computer Interaction - INTERACT 2013. 2013. pp. 651-658.

[19]J. Famaey, S. Latré, J. Strassner, and F. De Turck, "Semantic context dissemination and service matchmaking in future network management," International Journal of Network Management, vol. 22, no. 4, pp. 285- 310, Jul. 2012.

[20]M. Steenhuyse, J. Hoebeke, A. Ackaert, I. Moerman, and P. Demeester, "TV-kiosk: An open and extensible platform for the wellbeing of an ageing population," in Grid and Pervasive Computing Workshops, ser. Lecture Notes in Computer Science. Springer Berlin Heidelberg, 2012, vol. 7096, pp. 54-63.

[21]B. Frank, Z. Shelby, K. Hartke, and C. Bormann, "Constrained application protocol (CoAP)," 
Working Draft, IETF Secretariat, Fremont, CA, USA, Tech. Rep. draft-ietf-core-coap-11.txt, Dec. 2012.

\section{FIGURES}

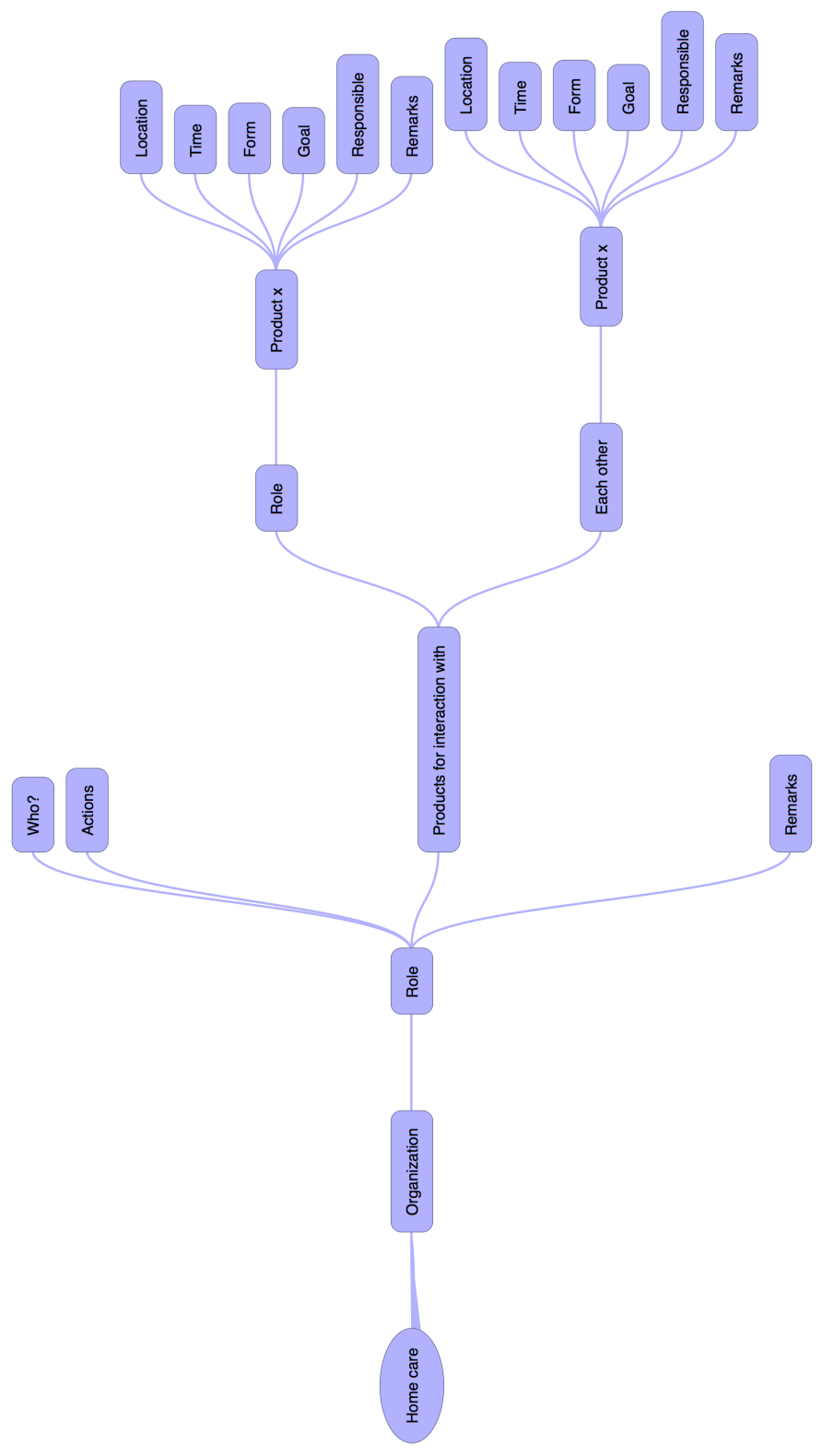




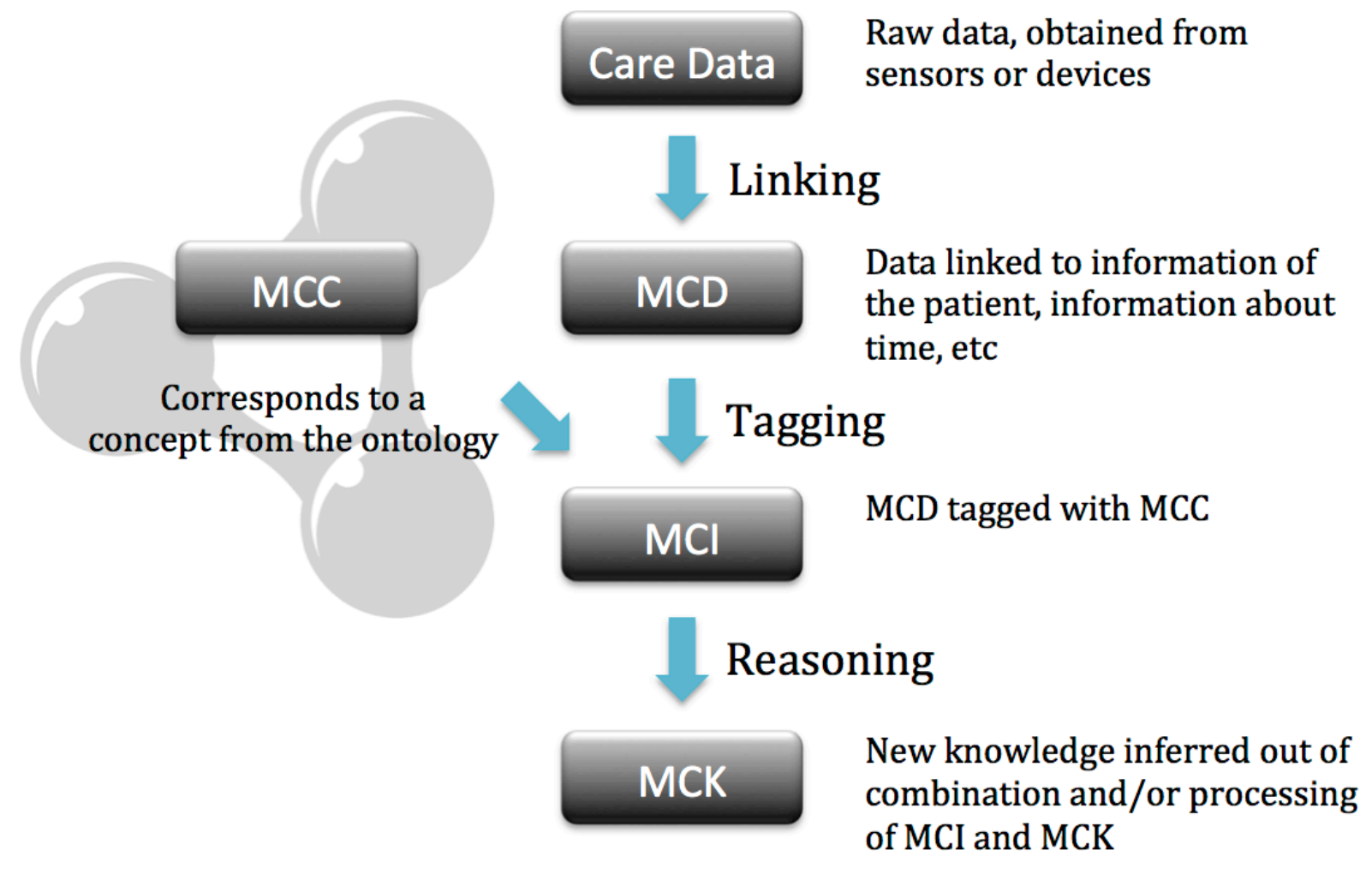

FIGURE 2 - VISUALIZATION OF THE DEPENDENCIES AND DEFINITIONS OF THE OCCS TERMS

Raw data, also called Care Data $(C D)$, originates from the patient's residence through a various range of sensors. Next, CD is linked to information about the patient and enriched with timestamps, transforming it into Meta Care Data (MCD). Following, tags are added to MCD which results in Meta Care Information (MCl). This tagging process can be done in two ways, either tagging by Meta Care Concepts, originating form the ontology, or with some plain nouns (that will be translated into proper MCCs later on). Meta Care Knowledge (MCK) is inferred out of the combination and/or processing of both $\mathrm{MCl}$ and $\mathrm{MCK}$. 


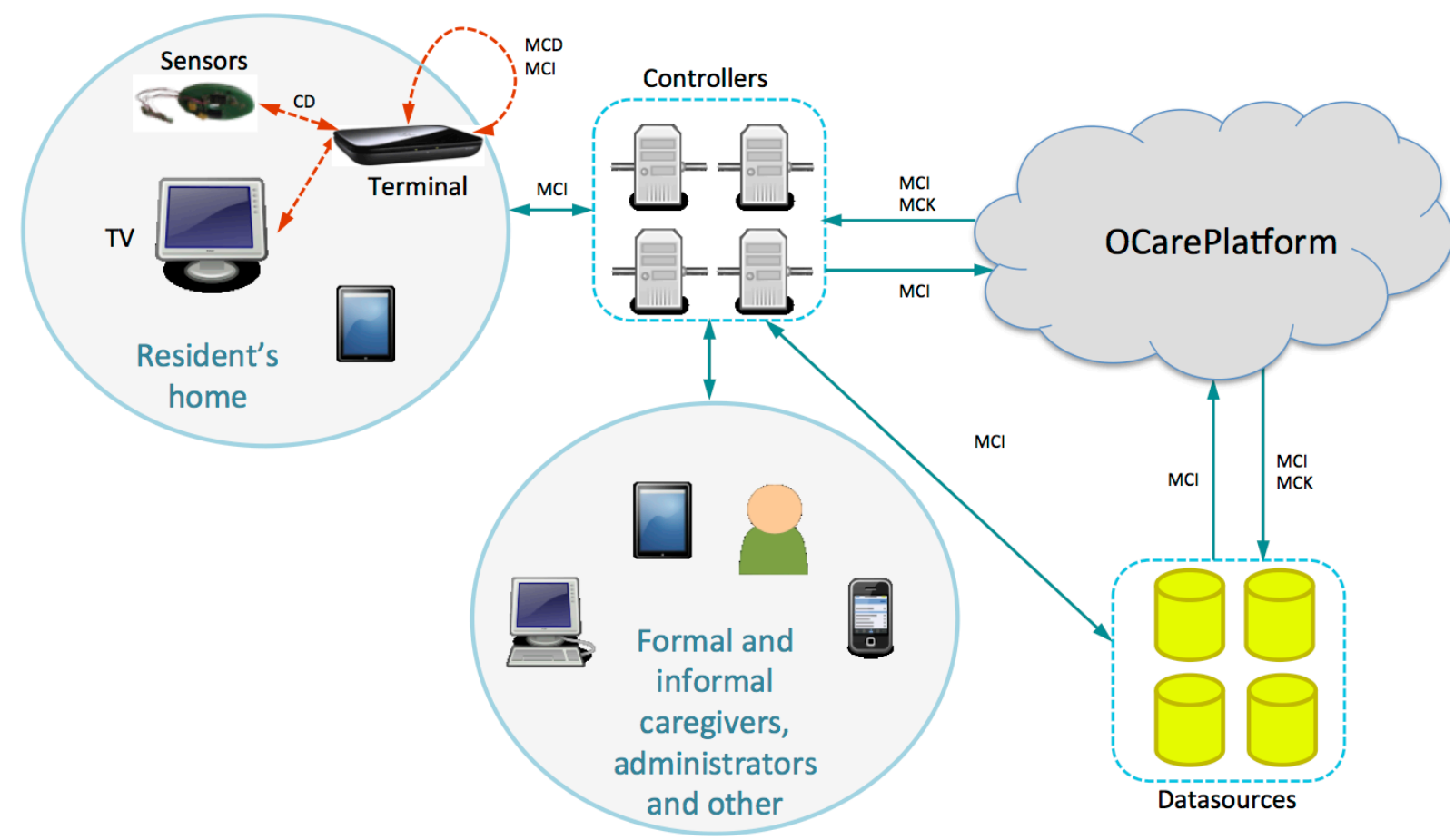

FIGURE 3 - GENERAL VIEW ON THE ARCHITECTURE OF THE OCCS SYSTEM

$C D$ is generated by the television and sensors, residing in the patient's home. $C D$ is collected by the terminal, where it will be first translated into $\mathrm{MCD}$ and next into $\mathrm{MCl}$. Following, $\mathrm{MCl}$ is sent to the Controllers, a server-side system, managing the connection between the different clients and the OCarePlatform. The Controllers are also responsible for back-ups and storing the data within the Datasources. The OCarePlatform will infer new knowledge out of the $\mathrm{MCl}$ and generated $\mathrm{MCK}$, which will be sent to the Controllers. They will take care of the communication and representation of this information to the patient (in the home), formal and informal caregivers, administrators or other individuals. 


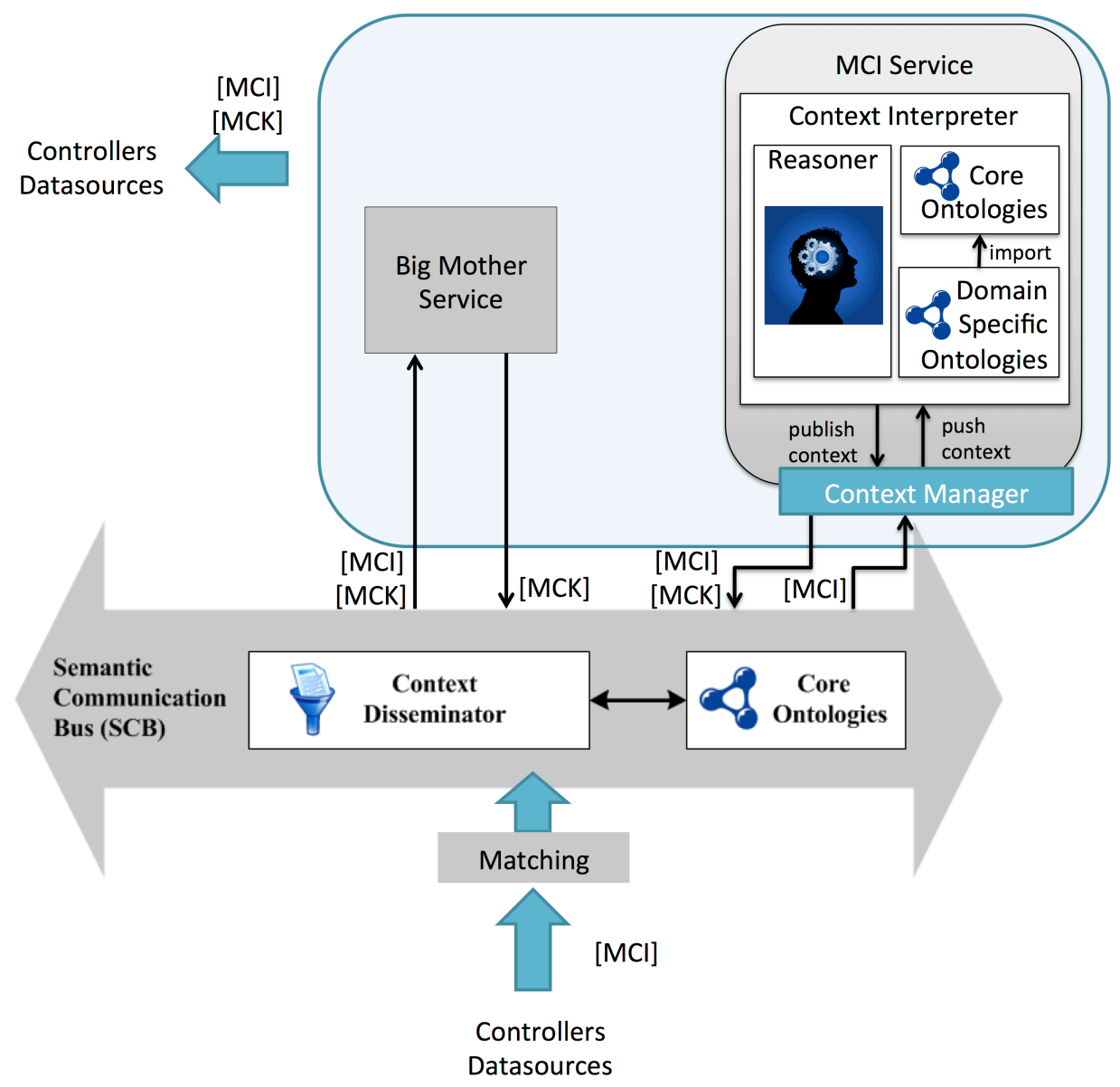

FIGURE 4 - CLOSE-UP OF THE OCAREPLATFORM

$\mathrm{MCl}$ will enter the OCarePlatform from the Controllers and Datasources through the Matching service. This Matching service is responsible for the translation of $\mathrm{MCl}$, which is tagged with nouns instead of MCC. Next, the data is passed to the Semantic Communication Bus (SCB). The SCB will process data and forward it to $\mathrm{MCl}$ services that have indicated they have an interest for this specific type of data. The SCB uses the continuous care ontology to interpret data and $\mathrm{MCl}$ services can register themselves through the Context Disseminator.

$\mathrm{MCl}$ Services can register themselves to the SCB through the Context Manager. This Manager is also responsible for the publishing of $\mathrm{MCK}$ and $\mathrm{MCl}$ as a result of the reasoning, The Context Interpreter preforms the reasoning, based on rules and a subset of the continuous care ontology (used within the SCB).

The Big Mother Service (BMS) is an intelligent software service, which implement rule-based algorithms to reason and combine $\mathrm{MCl}$ and $\mathrm{MCK}$ to come to more general context than the $\mathrm{MCl}$ services. 